EPJ Web of Conferences 49, 02005 (2013)

DOI: 10.1051/epjconf/20134902005

(C) Owned by the authors, published by EDP Sciences, 2013

\title{
Summary of Physics Results from the TOTEM Experiment
}

\author{
Giuseppe Latino ${ }^{1, a}$, on behalf of TOTEM Collaboration. \\ ${ }^{1}$ Department of Physics, Via Roma 56 - 53100 Siena. Università degli Studi di Siena and Gruppo Collegato INFN di Siena, Italy.
}

\begin{abstract}
The TOTEM experiment has performed several measurements related to its physics program in dedicated (high $\beta^{*}$, low $\mathcal{L}$ ) LHC fills at $\sqrt{s}=7 \mathrm{TeV}$. Under various beam and background conditions, the differential elastic (as a function of $|\mathrm{t}|$ ), elastic, inelastic and total pp cross-sections have been measured. A measurement of the forward charged particle $\eta$ density has also been performed. A summary of these measurements is here reported, as well as the first results from runs at the LHC energy of $\sqrt{s}=8 \mathrm{TeV}$.
\end{abstract}

\section{Introduction}

TOTEM [1] is one of the six experiments that investigate the frontier of high energy physics at the LHC. It has been designed for a precise measurement of the total $p p$ cross-section $\left(\sigma_{t o t}\right)$ down to $1 \div 2 \%$, for the study of the nuclear elastic $p p$ differential cross-section $\left(d \sigma_{e l} / d t\right)$ over a wide range of the squared four-momentum transfer $|t| \sim(p \theta)^{2}$, as well as for the study of inelastic and diffractive processes (to be performed partially in cooperation with CMS). In particular, TOTEM is able to measure $\xi-(\equiv \Delta p / p),|t|-$ and mass- distributions for the intact ("leading") protons produced in di ffractive events, with acceptances depending on the beam optics.

Given the lack of a complete description, based on a solid theoretical ground in the framework of the QCD, for the "soft" hadron interactions, many details of di ffractive (due to color singlet exchange) and non-diffractive (due to color exchange) processes are still poorly understood. At the same time these processes, with close links to proton structure and low energy QCD, represent a big fraction of $\sigma_{\text {tot }}$. Consequently, the experimental measurements performed by TOTEM are very important because they represent a reference for the phenomenological models describing the soft hadron interactions.

Furthermore, the study of the charged particle production in the forward region provides a significant contribution to the understanding of cosmic ray (CR) physics, heavily based on the simulation of primary CR particle interactions with the atmosphere. The existing models are indeed characterized by significant inconsistencies in the forward region for the predictions on energy flow, multiplicity and other quantities related to cosmic ray air showers. Thanks to its forward trackers TOTEM is able to perform the measurements of the charged particle pseu-

\footnotetext{
a e-mail: giuseppe.latino@pi.infn.it
}

dorapidity ${ }^{1}$ density $\left(\mathrm{dN}_{c h} / d \eta\right)$ and multiplicity distribution $\left(\mathrm{N}_{c h}\right)$ in a previously unexplored forward $\eta$ region.

Finally, a common data taking of TOTEM with CMS (resulting in the largest acceptance detector ever built at a hadron collider) opens the possibility of more detailed studies on inelastic processes, including hard diffraction [2].

After a brief description of the detector apparatus, a summary of the current TOTEM physics results is given in this paper.

\section{Detector setup}

In order to fulfill its physics program TOTEM has to cope with the challenge of triggering and recording events in the very forward region with a good acceptance for particles produced at very small angles with respect to the beam. This involves the detection of elastically scattered and diffractive protons at a location very close to the beam, together with efficient forward charged particle detection in inelastic events with losses reduced to few per-cents. This is accomplished with its experimental setup, characterized by three detector components located on both sides of the interaction point (IP) 5 , shared with CMS (figure 1): the T1 and T2 inelastic telescopes, embedded inside the forward region of CMS, and the "Roman Pots" detectors, placed on the beam-pipe of the outgoing beams in two stations at about $147 \mathrm{~m}$ and $220 \mathrm{~m}$ from IP 5 .

Charged track reconstruction in the $3.1<|\eta|<6.5$ range is provided by the $\mathrm{T} 1$ and $\mathrm{T} 2$ gas detectors, with a $2 \pi$ coverage and with a very high efficiency [3]. They allow the measurement of inelastic rates with small losses, given their trigger capability with a geometric acceptance grater than $95 \%$ for all inelastic events. At the same time they can provide the reconstruction of the interaction vertex, allowing to reject background events.

\footnotetext{
${ }^{1}$ The pseudorapidity $\eta$ is defined as: $\eta=-\ln \left(\tan \frac{\theta}{2}\right)$, where $\theta$ is the polar angle of the particle direction with respect to the counterclockwise beam direction.
} 


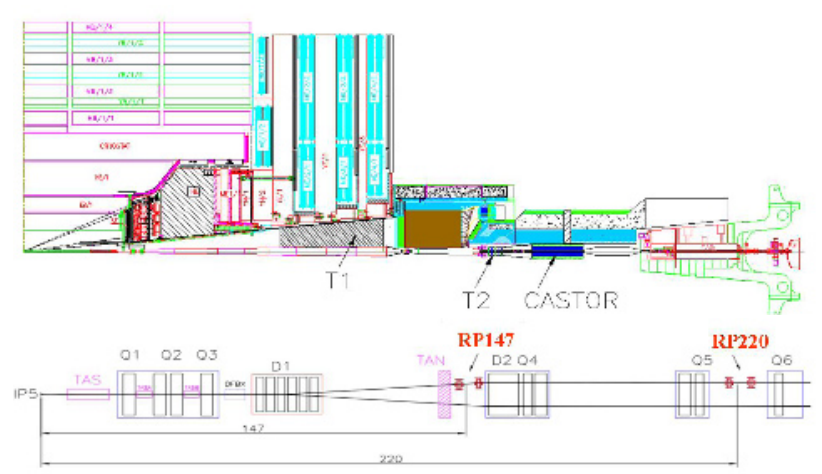

Figure 1. Top: T1 and T2 location in the forward region of CMS. Bottom: Roman Pots location along the LHC beam-line. All TOTEM detectors are installed on both sides of IP 5 .

Each arm of the T1 telescope, located at $\sim 9 \mathrm{~m}$ from IP 5, covers the range $3.1<|\eta|<4.7$ and is composed of five planes of "Cathode Strip Chambers" (CSC) [1] (figure 2, left). Each plane consists of six trapezoidal CSCs, with $10 \mathrm{~mm}$ thick gas gap and a gas mixture of $\mathrm{Ar} / \mathrm{CO}_{2} / \mathrm{CF}_{4}(40 \% / 50 \% / 10 \%)$, providing the measurement of the charged particle hit position with a spatial resolution of $\sim 1 \mathrm{~mm}$. The anode wires (pitch of $3 \mathrm{~mm}$ ), also giving level-1 trigger information, are parallel to the trapezoid base, while the cathode strips (pitch of $5 \mathrm{~mm}$ ) are rotated by $\pm 60^{\circ}$ with respect to the wires.

The $\mathrm{T} 2$ telescope extends charged track reconstruction to the range $5.3<|\eta|<6.5$ [1]. It is based on the "Gas Electron Multiplier" (GEM) technology [4], which has been chosen thanks to its good spatial resolution, excellent rate capability and good resistance to radiation. Each T2 halfarm, located at $\sim 13.5 \mathrm{~m}$ from IP 5, is made by the combination of ten aligned detectors planes having an almost semicircular shape (figure 2, right). The T2 GEMs are characterized by a triple-GEM structure with a gas mixture of $\mathrm{Ar} / \mathrm{CO}_{2}(70 \% / 30 \%)$ [5]. The read-out board has two separate layers with different patterns: one with $256 \times 2$ concentric circular strips ( $80 \mu \mathrm{m}$ wide, pitch of $400 \mu \mathrm{m})$, allowing the track radial coordinate reconstruction with a resolution of $\sim 100 \mu \mathrm{m}$; the other with a matrix of $24 \times 65$ pads (from $2 \times 2 \mathrm{~mm}^{2}$ to $7 \times 7 \mathrm{~mm}^{2}$ in size) providing the reconstruction of the track azimuthal coordinate with a resolution of $\sim 1^{\circ}$, as well as level-1 trigger information.
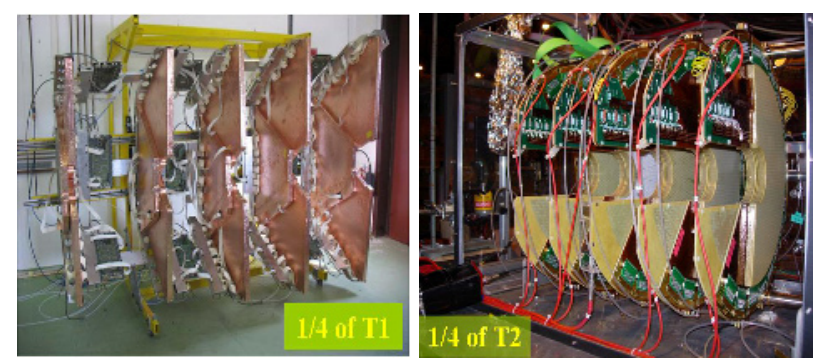

Figure 2. Left (right): one half-arm of the T1 (T2) telescope.
'Leading" protons with a scattering angle down to few $\mu \mathrm{rad}$ are detected by special movable beam-pipe insertions called Roman Pots (RPs). They host silicon detectors which are moved very close to the beam, when it is in stable conditions. As the detection of protons at such small scattering angles requires a detector active area as close to the beam as $\sim 1 \mathrm{~mm}$, a novel "edgeless planar silicon" detector technology has been developed for TOTEM RPs in order to have an edge dead zone minimized to only $\sim 50 \mu \mathrm{m}$ [6]. Each RP station is composed of two units, in order to have a lever harm for a better local track reconstruction and a higher efficiency of trigger selection by track angle. Each unit consists of three pots, two vertical and one horizontal completing the acceptance for diffractively scattered protons. Each pot contains a stack of 10 planes of silicon strip detectors (figure 3). Each plane has 512 strips (pitch of $66 \mu \mathrm{m})$, oriented at $+45^{\circ}$ (5 " $u$ "-planes) or at $-45^{\circ}$ ( $5 " v "$-planes) w.r.t. the detector edge facing the beam, allowing a single hit resolution of $\sim 10 \mu \mathrm{m}$.

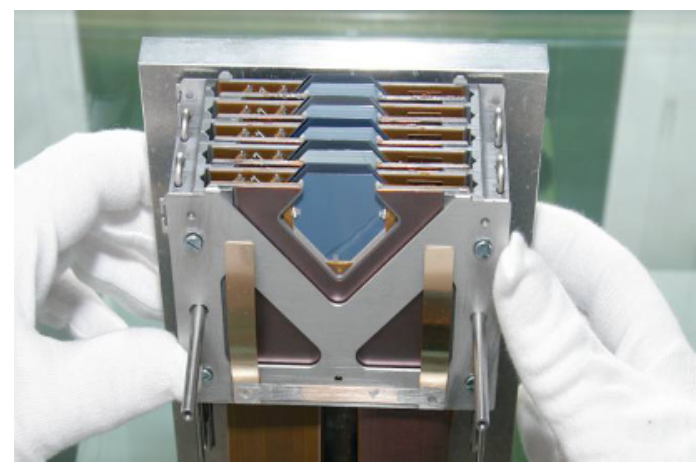

Figure 3. Silicon detectors hosted in one pot.

The VFAT digital chip [3], specifically designed for TOTEM and characterized by trigger capabilities, provides the read-out of all TOTEM sub-detectors and the related trigger information.

\section{Measurements at $\sqrt{s}=7 \mathrm{TeV}$}

A precise measurement of small scattering angles for the protons (needed to access small $|t|$ values) requires, besides a close approach of the detectors to the outgoing beams, the beam angular divergence $\left(\sigma_{\Theta}^{*}\right)$ to be as small as possible, at the level of few $\mu$ rads. Since $\sigma_{\Theta}^{*}=\sqrt{\epsilon / \beta^{*}}$, this can be obtained either by increasing the value of the betatron function $\beta$ at IP $\left(\beta^{*}\right)$ or by reducing the beam emittance $(\epsilon)$. Given the higher control on $\beta^{*}$, special LHC runs with a dedicated machine optics configuration at high $\beta^{*}$ are required. The consequent increase in beam size at the interaction point characterizes these runs with a low luminosity $(\mathcal{L})$, which is anyway needed in TOTEM analyses in order to avoid extra interactions between the colliding bunches. The data taken during 2010 and 2011 in special runs with various LHC configurations allowed the first TOTEM measurements, as reported in the following. 


\subsection{Elastic scattering}

TOTEM has performed a first measurement of $d \sigma_{e l} / d t$ in the $0.36<|t|<2.5 \mathrm{GeV}^{2}$ range using data taken in 2010 with the standard LHC optics $\left(\beta^{*}=3.5 \mathrm{~m}\right)$ during a dedicated run at low luminosity [7]. A total luminosity of 6.1 $\mathrm{nb}^{-1}$ was integrated, with the RP detectors approaching the beams as close as 7 times the transverse beam size $\left(\sigma_{b}\right)$. This measurement has been extended to $|t|$ values as low as $2 \cdot 10^{-2} \mathrm{GeV}^{2}$ by analyzing the data recorded in a short run taken in June 2011 with a dedicated beam optics configuration ( $\beta=90 \mathrm{~m}$ ) and low luminosity, during which a luminosity of $1.7 \mu \mathrm{b}^{-1}$ was integrated with the RPs placed at $10 \sigma_{b}$ [8]. This made for the first time the extrapolation to the optical point $(t=0)$ possible, so allowing the determination of the elastic scattering cross-section $\left(\sigma_{e l}\right)$ as well as of $\sigma_{\text {tot }}$ via the optical theorem. Even smaller $|t|$ values, down to $5 \cdot 10^{-3} \mathrm{GeV}^{2}$, were then obtained with an updated analysis performed using different higher statistics data sets (for a total of $83 \mu \mathrm{b}^{-1}$ ) taken in October 2011 with special runs at $\beta^{*}=90 \mathrm{~m}$, where the RPs were put very close to the beam ( 4.8 to $6.5 \sigma_{b}$ ) [9]. This enabled the observation of $91 \%$ of $\sigma_{e l}$, to be compared to only $67 \%$ of the previous measurement, which gave an improved extrapolation to the optical point. The data taking and analysis strategies were substantially the same for all these measurements.

The background was significantly reduced a the trigger level by requiring trigger tracks on both sides of the IP. A high trigger efficiency (over $99 \%$ per proton) was achieved by using a loose trigger requiring a track segment in any of the vertical RPs of the $220 \mathrm{~m}$ stations. Elastic candidates were then selected offline by requiring a reconstructed track in both ( $u$ - and $v$-) projections of the vertical RP units on each side of the IP in a "diagonal" topology: top (bottom) left of IP - bottom (top) right of IP.

Using the "optical functions", which describe the explicit proton path from the IP to the RPs location through the LHC magnet elements as a function of the proton position $\left(x^{*}, y^{*}, z^{*}=0\right)$ and scattering angle $\left(\Theta_{x}^{*}, \Theta_{y}^{*}\right)$ at the IP, the horizontal $\left(\Theta_{x}^{*}\right)$ and vertical $\left(\Theta_{y}^{*}\right)$ projections of the scattering angle are deduced from the measurement of the proton position at the RPs $(x, y)$ according to the following equations:

$$
x=L_{x} \Theta_{x}^{*}+v_{x} x^{*}, \quad y=L_{y} \Theta_{y}^{*}+v_{y} y^{*},
$$

where $L_{x, y}$ and $v_{x, y}$ represent the optical functions at the RPs position. Being related to the betatron function, they depend on the LHC optics configuration and define the related $|t|$-range acceptance of the RPs. With the standard $\left(\beta^{*}=3.5 \mathrm{~m}\right)$ and intermediate $\left(\beta^{*}=90 \mathrm{~m}\right)$ optics the vertical scattering angle $\Theta_{y}^{*}$ can be directly reconstructed from the track position $y$, whereas the horizontal component $\Theta_{x}^{*}$ is optimally reconstructed from the local track angle $\Theta_{x}=$ $d x / d s$ at the RPs.

Dedicated procedures have been performed in order to ensure the precision and the reproducibility of all RP detector planes alignment with respect to each other and to the position of the beam center, one of the most delicate ad difficult tasks of the experiment. A precise relative alignment (at the level of few $\mu \mathrm{m}$ ) of all three RPs in a unit has been obtained during the data analysis by correlating their position via common particle tracks reconstruction in the overlap zone of the horizontal RPs with the vertical ones. The global symmetrical alignment of all the RPs with respect to the beam center has been obtained (with a precision of $\sim 20 \mu \mathrm{m}$ ) during dedicated beam fills by moving them towards the sharp beam edge cut by the beam collimators, until a beam losses spike is observed downstream of the RPs. A final horizontal and vertical alignment finetuning has then been achieved from studies on the reconstructed tracks by exploiting the azimuthal symmetry of elastic scattering.

Given the collinearity of the elastically scattered protons the event selection has been performed by requiring a strict correlation (consistent with the beam divergence) between the two $\Theta_{x}^{*}$ (and $\Theta_{y}^{*}$ ) projections, expected to be the same on both sides of IP. Other cuts on the reconstructed kinematic variables have also been applied in order to remove diffractive (low $\xi$ cuts) and beam halo (vertex cuts) background. Acceptance limitations have been accounted for by assuming azimuthal symmetry of the elastic scattering and by correcting for smearing around the detector edges. Bin migration effects, due to angular resolution (related to detector resolution and beam divergence effects), have been corrected using a Monte Carlo (MC) based iterative procedure. The RPs trigger efficiency (>99.8\% @ 68\% CL) was evaluated by using the "zerobias" (trigger on bunch crossing) data stream. DAQ and reconstruction inefficiencies were calculated from dedicated studies on data. The total luminosity associated to the collected data has been derived from the instantaneous luminosity measured by CMS with an uncertainty of $4 \%$, integrated over the data taking period. The systematic uncertainty was found to be dominated by the luminosity uncertainty for $|t|<0.2 \mathrm{GeV}^{2}$ and by t-dependent (opticsrelated) contributions elsewere.

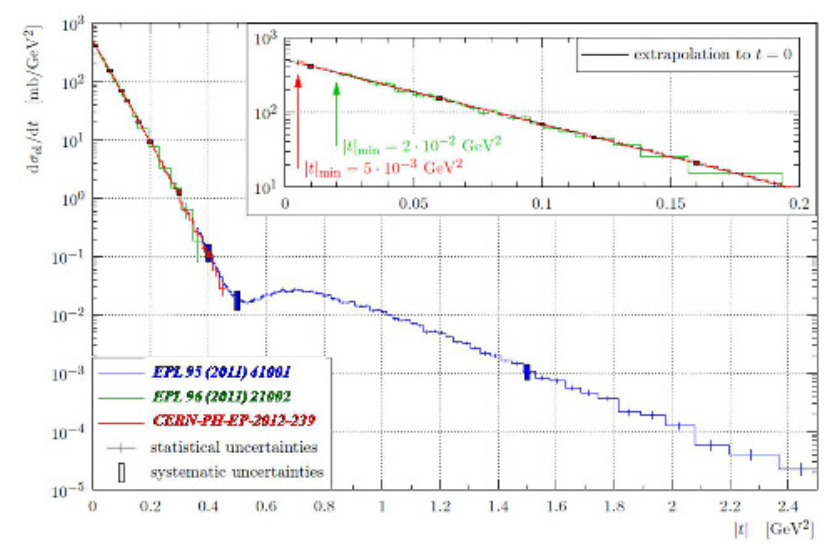

Figure 4. $d \sigma_{e l} / d t$ measurements performed by TOTEM in three different run conditions and RPs approaches to the beam. Each measurement is shown in a different color. On the right-top a zoom of the low $|t|$ region used for the extrapolation to $t=0$ is shown. 
All TOTEM differential cross-section measurements are given in figure 4 , where the right-top insert shows a zoom of the region used for the extrapolation to $t=0$ in the two measurements at $\beta^{*}=90 \mathrm{~m}$. For $|t|<0.2 \mathrm{GeV}^{2}$ the data can be described very well by an exponential function with slope $B=(19.9 \pm 0.3) \mathrm{GeV}^{-2}$ which, compared to previous collider experiments a lower energies, shows a steadily increasing value with the collision energy $\sqrt{s}$. The expected diffractive minimum, typically pronounced in pp scattering, is then observed at $|t|=(0.53 \pm 0.01)$ $\mathrm{GeV}^{2}$ and it is closer to $t=0$ respect to the measurements performed at lower energies. At higher $|t|$ the $d \sigma_{e l} / d t$ can be described by a power law $|t|^{-n}$ with $n=7.8 \pm 0.3$, in the $|t|$-range $1.5-2.0 \mathrm{GeV}^{2}$. The comparison with the predictions of different available theoretical models shows a partial consistency with the data (slope $B$, dip position and exponent $n$ at large $|t|$ ) only for some of them [7].

The low $|t|$ values reached with the $\beta^{*}=90 \mathrm{~m}$ optics made the exponential extrapolation to $t=0\left(\left.\frac{d \sigma_{e l}}{d t}\right|_{t=0}\right)$ possible, allowing to derive (by integration of the elastic distribution) the elastic cross section. In particular, the measurement performed at lower $|t|$ (down to $5 \cdot 10^{-3} \mathrm{GeV}^{2}$ ) gave the most precise extrapolation to the optical point, so the best $\sigma_{e l}$ measurement. The $\sigma_{e l}$ results obtained with the two different data sets are reported in table 1.

The optical theorem, relating the total to the elastic pp cross-section, was then used to obtain $\sigma_{\text {tot }}$ and the inelastic cross-section $\left(\sigma_{\text {inel }}\right)$ according to:

$$
\sigma_{t o t}^{2}=\left.\frac{16 \pi(\hbar c)^{2}}{1+\rho^{2}} \cdot \frac{d \sigma_{e l}}{d t}\right|_{t=0}, \quad \sigma_{\text {inel }}=\sigma_{t o t}-\sigma_{e l},
$$

where for the $\rho$ parameter ${ }^{2}$ was chosen the COMPETE preferred-model extrapolation of $0.141 \pm 0.007$ [10]. The errors on this measurement are dominated by the uncertainties related to the luminosity and to the extrapolation to $t=0$. The results obtained with the two different data sets are summarized in table 1 .

\subsection{Inelastic cross section}

Based on the same data taking as in reference [9] $\sigma_{\text {inel }}$ was also directly measured using inelastic events triggered by the $\mathrm{T} 2$ telescope and complemented by $\mathrm{T} 1$ telescope data, with the luminosity determination being provided by CMS [11].

In order to perform an optimized study of the trigger efficiency and of the beam-gas background corrections, the inelastic events were separated into three subsamples: with tracks in both T2 arms (mainly due to non diffractive and to double diffractive processes) and with tracks in only one ("+" or "-") of the two T2 arms (mainly due to single diffractive processes). Special emphasis was given to properly tune the simulation of the forward region, in particular in front and around $\mathrm{T} 2$, since the secondary particles, produced in this zone by the interaction of primary particles with the material, enhance the observed inelastic

${ }^{2} \rho=\Re\left|f_{e l}(0)\right| / \mathfrak{I}\left|f_{e l}(0)\right|$, where $f_{e l}(0)$ is the forward nuclear elastic amplitude. rate. To obtain the true inelastic rate the observed one had to be properly corrected. The corrections, derived with studies on proper MC samples, "zero bias" and "non colliding bunch" trigger data, were done in three steps allowing to get: the "T2 visible" cross-section $\left(\sim 95 \%\right.$ of $\sigma_{\text {inel }}$, related to events with at least one particle in the $|\eta|$ acceptance of T2, i.e. with $5.3<|\eta|<6.5$ ); the "global visible" cross-section (related to events with at least one particle up to the $|\eta|$ acceptance of T2, i.e. with $|\eta|<6.5$ ); the "missing" cross-section (related to events with only particles beyond the T2 acceptance, i.e. with $|\eta|>6.5$, mainly due to single diffractive processes with masses below $\sim$ $3.4 \mathrm{GeV} / \mathrm{c}^{2}$ ). These studies showed in particular a very good performance of the $\mathrm{T} 1$ and $\mathrm{T} 2$ detectors, characterized by an event reconstruction efficiency of about $98 \%$ (99\%) for an individual T1 (T2) arm. After comparing several MC models, the correction for the missing low mass single diffractive cross-section has been obtained based on the QGSJET-II-03 generator, which can well describe the measurements of low mass diffraction at lower energies. This correction $(4.2 \pm 2.1 \%)$ is the largest one to the inelastic rate and, given the large uncertainty, represents the second largest systematic uncertainty of this measurement after the one related to the luminosity (4\%). The result, reported in table 1 , is well in agreement with the previous TOTEM measurements deduced from the elastic scattering via the optical theorem [8,9] as well as compatible with the measurements performed by ALICE [12], ATLAS [13] and CMS [14]. However, given the unique $\eta$ coverage of the $\mathrm{T} 1$ and $\mathrm{T} 2$ detectors allowing to reach the lowest diffractive masses $\left(\mathrm{M}_{X}>3.4 \mathrm{GeV} / \mathrm{c}^{2}\right)$, this measurement is characterized by a minimal model dependence for the required low mass diffraction corrections respect to the measurements performed by the other LHC experiments.

Furthermore, an estimate of low mass diffraction contribution was obtained by comparing the full inelastic cross-section measurement of reference [9] (by construction derived without any assumption about low mass diffraction), with the direct "global visible" $(|\eta|<6.5$, or $\mathrm{M}_{X}>3.4 \mathrm{GeV} / \mathrm{c}^{2}$ ) inelastic cross-section measurement. From their difference a "missing" inelastic cross-section of $2.6 \pm 2.2 \mathrm{mb}$ was obtained, corresponding to an upper limit of $6.3 \mathrm{mb}$ at $95 \% \mathrm{CL}$ for diffractive events with $\mathrm{M}_{X}$ $<3.4 \mathrm{GeV} / \mathrm{c}^{2}$.

\subsection{Luminosity independent total cross-section}

A " $\rho$-independent" determination of $\sigma_{\text {tot }}$ has been obtained by summing directly the elastic [9] and the inelastic [11] cross sections:

$$
\sigma_{t o t}=\sigma_{e l}+\sigma_{i n e l}
$$

This result, reported in table 1 , is characterized by a larger uncertainty due to the direct propagation of the luminosity uncertainty.

However, the dependence of the $\sigma_{\text {tot }}$ measurement on the luminosity can be eliminated using the optical theo- 
Table 1. Summary of TOTEM elastic, inelastic and total cross-section measurements at $\sqrt{s}=7 \mathrm{TeV}$. The first two lines refer to the "elastic only" measurements (eq. 2) related to the June 2011 [8] and October 2011 [9] data taking periods. The third and fourth lines report respectively the $\rho$-independent (eq. 3) $[9,11]$ and the $\mathcal{L}$-independent (eq. 4) [15] measurements for the October 2011 data.

\begin{tabular}{llll}
\hline Data analysis & $\sigma_{e l}(\mathrm{mb})$ & $\sigma_{\text {inel }}(\mathrm{mb})$ & $\sigma_{\text {tot }}(\mathrm{mb})$ \\
\hline [8] & $24.8 \pm 1.2$ & $73.5 \pm 1.6$ & $98.3 \pm 2.8$ \\
[9] & $25.4 \pm 1.1$ & $73.2 \pm 1.3$ & $98.6 \pm 2.2$ \\
[9] [11] & $25.4 \pm 1.1$ & $73.7 \pm 3.4$ & $99.1 \pm 4.3$ \\
[15] & $25.1 \pm 1.1$ & $72.9 \pm 1.5$ & $98.0 \pm 2.5$ \\
\hline
\end{tabular}

rem:

$$
\sigma_{t o t}=\frac{16 \pi(\hbar c)^{2}}{1+\rho^{2}} \cdot \frac{d N_{e l} /\left.d t\right|_{t=0}}{N_{e l}+N_{\text {inel }}},
$$

where $N_{e l}$ and $N_{\text {inel }}$ represent respectively the elastic and inelastic rates integrated over a given data taking period. Using the above equation, the elastic and inelastic measurements performed (independently but simultaneously) in the same analyses of reference [9] and [11] respectively, were combined to obtain $\sigma_{\text {tot }}$ without taking into account the luminosity [15]. Furthermore, also $\sigma_{e l}$ and $\sigma_{\text {inel }}$ were derived independently from the luminosity using the measured ratio $N_{\text {el }} / N_{\text {inel }}$. The errors on this measurement are dominated by the uncertainties on the extrapolation to $t=0$ and on the correction for the contribution from low mass diffraction. The cross-sections measured with this “ $\mathcal{L}$-independent" method, reported in table 1 , are well in agreement with the previous TOTEM measurements, which confirms the understanding of the systematic uncertainties and of the corrections applied in the different analysis strategies. These measurements are also reported in figure 5, showing a very good agreement with the expectations from the overall fit of previously measured data over a large range of energies [10].

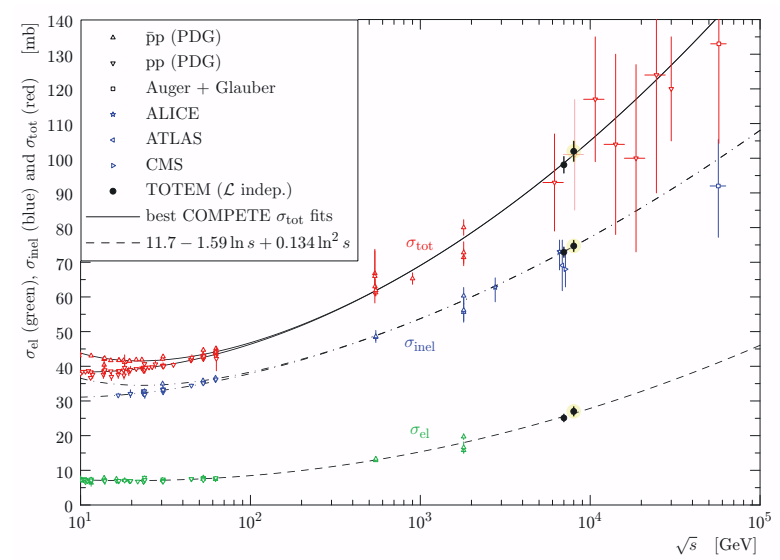

Figure 5. Compilation of the $\sigma_{\text {tot }}, \sigma_{\text {inel }}$ and $\sigma_{e l}$ measurements. The black points show the $\mathcal{L}$-independent measurements performed by TOTEM at $\sqrt{s}=7$ and $\sqrt{s}=8 \mathrm{TeV}$. The continuous black lines (lower for $p p$, upper for $p \bar{p}$ ) represent the best fit to $\sigma_{\text {tot }}$ data by the COMPETE Collaboration [10], while the dashed line is related to a fit to the $\sigma_{e l}$ data. The dash-dotted lines, corresponding to $\sigma_{\text {inel }}$, are derived as the difference between the two previous fits.
The optical theorem can also be applied in a complementary way in order to obtain the integrated luminosity $\left(\mathcal{L}_{\text {int }}\right)$ for a given data taking period:

$$
\mathcal{L}_{i n t}=\frac{1+\rho^{2}}{16 \pi(\hbar c)^{2}} \cdot \frac{\left(N_{e l}+N_{\text {inel }}\right)^{2}}{d N_{e l} /\left.d t\right|_{t=0}}
$$

Thus, the elastic and inelastic rates were also combined to obtain an absolute (and independent) $\mathcal{L}_{\text {int }}$ measurement. The TOTEM results were found to be well in agreement with the CMS ones within their uncertainties $(\sim 4 \%$ for both) [15].

\subsection{Forward charged particle pseudorapidity density}

A measurement of the pseudorapidity density $\left(\mathrm{dN}_{c h} / \mathrm{d} \eta\right)$ of forward charged particles was also performed by TOTEM using the data taken in May 2011 with an inclusive T2 trigger during special LHC fills at low luminosity [16].

As a key aspect of this study is an optimal particle track reconstruction, a particular effort has been devoted in the analysis in order to correct for misalignment biases, found to be dominated by global displacements of the T2 quarters. The relative positions of the detector planes within a T2 quarter in terms of $x$ - and $y$-shifts (internal alignment) have been obtained using two different methods (iterative and MILLEPEDE), giving consistent results in the related corrections with an uncertainty of about 30 $\mu \mathrm{m}$. The relative alignment between the two neighbouring quarters of an arm has been derived using tracks reconstructed in the overlap regions. The global detector alignment respect to its nominal position (corrections for tilts and shifts), of main importance for the present analysis, has been achieved by exploiting the expected symmetry in the track parameters distributions, as well as the position on each T2 plane of the "beam pipe shadow" (a circularshaped zone characterized by a very low track efficiency due to primary particles interaction in the $\eta \sim 5.54$ beam pipe cone in front of T2). The combination of all these methods resulted in corrections for $x$ - and $y$-shifts with a precision of $\sim 1 \mathrm{~mm}$ and for $x z$ - and $y z$-plane tilts with a precision of $\sim 0.4 \mathrm{mrad}$.

Secondary track rejection is another important step of this analysis as about $80 \%$ of the $\mathrm{T} 2$ reconstructed tracks is due to secondaries. It has been performed with a procedure for the primary/secondary particle discrimination based on a proper track parameter, which has been found in detailed MC studies to be the most effective for this task and the most stable against misalignment errors. Primary track efficiency (found to be $\sim 80 \%$ ) and smearing effects corrections have been obtained from MC studies.

The results of the measurement, which refers to events with at least one charged particle with $P_{T} \geq 40 \mathrm{MeV} / \mathrm{c}$ and $\tau>0.3 \cdot 10^{-10}$ s going in the $\mathrm{T} 2$ acceptance region, are reported in figure 6 . Here the red squares show the experimental values in terms of the average of the results found with the four T2 quarters, with the error bars representing the combination of the statistical and systematic errors (up to $\sim 10 \%$ ), dominated by primary track efficiency and 
global alignment corrections uncertainties. Figure 6 also shows the comparison of some MC expectations with the TOTEM results. None of the theoretical models has been found to fully describe the data, the cosmic ray MC generators (SYBILL and QGSJET-II) showing a better agreement for the slope.

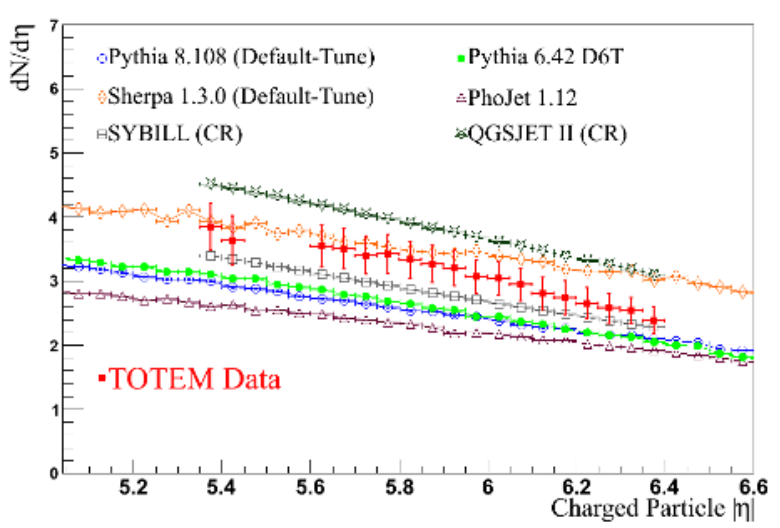

Figure 6. $\mathrm{dN}_{c h} / \mathrm{d} \eta$ distribution measured by Totem. The experimental results (red squares), obtained as the average of the four T2 quarters, are reported with the error bars including both statistical and systematic errors. The predictions from some MC generators are reported for comparison.

\section{Measurements at $\sqrt{s}=8 \mathrm{TeV}$}

TOTEM also took data at $\sqrt{s}=8 \mathrm{TeV}$ during 2012 in special LHC runs. In some of them the first common data taking with CMS was also performed, with a TOTEM $\Leftrightarrow$ CMS trigger exchange. The October 2012 run was in particular characterized by $\beta^{*}=1000 \mathrm{~m}$ and a RPs approach of $3 \sigma_{b}$ to the beam center, allowing to measure $\sigma_{e l} / d t$ down to $6 \cdot 10^{-4} \mathrm{GeV}^{2}$ so in principle opening the possibility of a determination of the $\rho$ parameter by studying the Coulomb-Nuclear interference region. Several analyses on these data are at the moment ongoing.

The data samples recorded on July 2012 during special fills with $\beta^{*}=90 \mathrm{~m}$ have been used to perform the $\mathcal{L}$-independent measurement of $\sigma_{\text {tot }}(101.7 \pm 2.9 \mathrm{mb}), \sigma_{e l}$ $(27.1 \pm 1.4 \mathrm{mb})$ and $\sigma_{\text {inel }}(74.7 \pm 1.7 \mathrm{mb})$ at $\sqrt{s}=8 \mathrm{TeV}$ [17]. These results, also reported in figure 5, are again in very good agreement with the expectations from the overall fit of previously measured data.

\section{Summary and Conclusions}

Using the data taken during 2010 and 2011 in special LHC runs with different beam conditions $\left(\beta^{*}=3.5\right.$ and $90 \mathrm{~m}$, low $\mathcal{L}$ ) the TOTEM experiment has performed its first measurements at $\sqrt{s}=7 \mathrm{TeV}$. These comprise the $d \sigma_{\text {el }} / d t$ measurement in a wide $|t|$ range $\left(5 \cdot 10^{-3}<|t|<2.5 \mathrm{GeV}^{2}\right)$, as well as the measurement of $\sigma_{e l}, \sigma_{\text {inel }}$ and $\sigma_{\text {tot }}$ using different methods. These last results are in good agreement within the errors with the theoretical expectations and, for what concerns $\sigma_{\text {inel }}$, with the measurements of other LHC experiments. The $\mathrm{dN}_{c h} / \mathrm{d} \eta$ distribution was also measured in the $5.3<|\eta|<6.4$ range.

The analysis of the data taken in 2012 at $\sqrt{s}=8 \mathrm{TeV}$ with $\beta^{*}=90 \mathrm{~m}$ gave the $\mathcal{L}$-independent measurement of $\sigma_{\text {tot }}$, $\sigma_{e l}$ and $\sigma_{\text {inel }}$, again found in good agreement with the theoretical predictions. Work is in progress for the analysis of other data samples taken at $\sqrt{s}=8 \mathrm{TeV}$. In particular, the ones with $\beta^{*}=1000 \mathrm{~m}$ are expected to open the possibility of the $\rho$ parameter measurement, while the ones taken jointly with CMS will provide the first common physics results on hard and soft diffraction as well as the $\mathrm{dN}_{c h} / \mathrm{d} \eta$ measurement over the whole $\eta$ range up to $|\eta| \sim 6.5$.

\section{Acknowledgements}

We gratefully thanks the Conference Organizers for their kind invitation to this very nice event in the wonderful town of Kyoto.

\section{References}

[1] V. Berardi et al. (TOTEM Collaboration), CERNLHCC-2004-002, CERN-LHCC-2004-020 (2004).

[2] M. Albrow et al. (The CMS and TOTEM DFP-WG), CERN-LHCC-2006-039/G-124 (2006).

[3] G. Anelli et al. (TOTEM Collaboration), JINST 3:S08007 (2008).

[4] F. Sauli, Nucl. Instrum. Methods A 386, 531 (1997).

[5] S. Lami et al., Nucl. Phys. B172, 231 (2007).

[6] E. Noschis et al., Nucl. Instrum. Methods A 563, 41 (2006).

[7] G. Antchev et al. (TOTEM Collaboration), Europhys.Lett. 95, 41001 (2011).

[8] G. Antchev et al. (TOTEM Collaboration), Europhys.Lett. 96, 21002 (2011).

[9] G. Antchev et al. (TOTEM Collaboration), CERNPH-EP-2012-239 (2012). Accepted for publication to Europhys.Lett. .

[10] J.R. Cudell et al. (COMPETE Coll.), Phys. Rev. Lett. 89, 201801 (2002).

[11] G. Antchev et al. (TOTEM Collaboration), CERNPH-EP-2012-352 (2012).

Accepted for publication to Europhys.Lett. .

[12] B. Abelev et al. (ALICE Collaboration), CERN-PHEP-2012-238, arXiv:1208.4968 (2012).

[13] G. Aad et al. (ATLAS Collaboration), Nature Commun. 2, 463 (2011).

[14] S. Chatrchyan et al. (CMS Collaboration), CMS-PAS-FWD-11-001, CERN-PH-EP-2012-293, arXiv:1210.6718 (2012).

[15] G. Antchev et al. (TOTEM Collaboration), CERNPH-EP-2012-353 (2012). Accepted for publication to Europhys.Lett..

[16] G. Antchev et al. (TOTEM Collaboration), Europhys.Lett. 96, 31002 (2012).

[17] G. Antchev et al. (TOTEM Collaboration), CERNPH-EP-2012-354 (2012).

Submitted for publication to Physical Review Letters. 